\title{
Comparative study of surgical site infection with or without post cesarean prophylactic oral antibiotics; a single-blinded randomized clinical trial
}

\author{
Farzaneh Broumand $^{1 \oplus}$, Naghmeh Zand Vakili ${ }^{2}$ Zahra Yekta $^{(\mathbb{D}}$, Shabnam Vazifekhah $^{1 *(\mathbb{D}}$ \\ ${ }^{1}$ Department of Obstetrics and Gynecology, School of Medicine, Shahid Motahari Hospital, Urmia University of Medical \\ Sciences, Urmia, Iran \\ ${ }^{2}$ Urmia University of Medical Sciences, Urmia, Iran \\ ${ }^{3}$ Department of Community Medicine, Urmia University of Medical Sciences, Urmia, Iran
}

\section{Correspondence to:}

Shabnam Vazifekhah,

Email: shabnamvazifekhah1985@

gmail.com,

vazifehkhah.s@umsu.ac.ir

Received: 19 July 2021

Accepted: 18 Oct. 2021

ePublished: 28 Oct. 2021

Keywords: Superficial infection Deep infection, Pelvic infection, Cesarean

\begin{abstract}
Introduction: Surgical site infection (SSI) is caused by cesarean section in the hospital and its prevalence in the studies is up to $16 \%$.

Objectives: This study aimed to compare the rate of infection in women undergoing cesarean section. Oral clindamycin and cefalexin were administered for 48 hours as prophylactic antibiotics.

Patients and Methods: In this clinical trial study, women with emergency cesarean section were divided into two groups. Intervention was oral administration of cefalexin 500mg every 6 hours and clindamycin 300 mg every 6 hours for 48 hours. All participants were referred to the gynecology center on day $7-10$, at the time of removal of the sutures, and within 30 days after cesarean section, to assess the presence or absence of wound infection after surgery during 30 days.

Results: In this clinical trial study, 462 pregnant women undergoing cesarean section were enrolled in the intervention and control groups. Of 231 patients in the intervention group, 15 women (6.5\%) had cesarean section infection (13 cases with superficial, and 2 cases with deep infection). In the control group, 45 cases $(19.5 \%)$ had cesarean section infection (31 cases with superficial, 10 cases with deep, and 4 cases with developed pelvic infection) $(P=0.001)$. Age, pre-cesarean length of stay, pre-term incision, type of incision, discharge longer than 18 hours after cesarean section, and maternal diabetes were significantly different regarding cesarean section infection in both groups. The frequency of cesarean section infection was less in the intervention group $(P<0.001)$.

Conclusion: Administration of prophylactic antibiotic can have a significant role in reducing cesarean section infection.

Trial Registration: Registration of trial protocol has been approved in Thailand registry of clinical trials (identifier: TCTR20201204002, http://www.clinicaltrials.in.th/index.php?tp=regtrials\&menu=trialsearch\&smenu=fulltext\&task=search\&task2=view1\&id=7120, ethical code; IR.UMSU.REC.1397.323).
\end{abstract}

Introduction

Cesarean section is a surgical procedure on the mother's abdomen and uterus to remove the fetus. Cesarean section is performed when the vaginal delivery is risky for both mother and fetus. Cesarean section can cause serious complications (1) such as wound infection, metritis (inflammation of the uterus) and pelvic infections. The prevalence of surgical site infection (SSI) in studies is up to $16 \%$. In developing countries, SSI affects two-thirds of patients undergoing any surgery (2). Even in developed countries, SSI is the most common nosocomial infection (14\%-16\%). Emergency cesarean section includes breech presentation, prolonged drainage and failure to respond to induction and history of cesarean section and other

\begin{abstract}
Key point
In a single-blinded randomized clinical trial as a comparative study of surgical site infection with or without post-cesarean prophylactic oral antibiotics, we found a significant difference regarding cesarean section infection between the two groups of use or nonuse of oral prophylactic antibiotics. Our study showed that the administration of prophylactic antibiotics plays an important role in reducing the incidence of cesarean section infection. It is recommended that the administration of prophylactic antibiotics be prescribed to mothers undergoing cesarean section.
\end{abstract}

reasons. Risk factors for SSI include chorioamnionitis, body mass index (BMI)> $35 \mathrm{~kg} / \mathrm{m}^{2}$, corticosteroid use, prolonged labor, non-use of prophylactic antibiotics, pre-gestational diabetes, length of operation $<38$ minutes, preeclampsia, hypertension,

Copyright $\odot 2022$ The Author(s); Published by Society of Diabetic Nephropathy Prevention. This is an open-access article distributed under the terms of the Creative Commons Attribution License (http://creativecommons.org/licenses/by/4.0), which permits unrestricted use, distribution, and reproduction in any medium, provided the original work is properly cited. 
duration of labor $<12$ hours, nulliparous, multiple births, premature rupture of membranes, gestational diabetes, previous cesarean section and other risk factors (3). Several methods have been suggested to control infection before surgery including preoperative prophylactic antibiotics such as intravenous cefazolin $1 \mathrm{~g}$ for 60 minutes before skin incision plus 500mg azithromycin as prophylactic antibiotics to reduce the risk of endometritis, and wound infection. Skin prep with chlorhexidine, or povidoneiodine, use of scissors instead of shaving the hair, vaginal prep with chlorhexidine before surgery or removal of the placenta by pulling the umbilical cord instead of removing by hand, rinsing the abdomen with saline and using a subcutaneous drain during surgery or dressing change within 24-48 hours and also the administration of chlorhexidine gluconate soap after removal of the dressing $(3,4)$. Infection of the organ or surrounding area and other evidence of deep wound infection can be seen on direct examination during surgery, pathology or radiographic images. Diagnosis by the attending surgeon or physician is based on at least two of the fever above 38 degrees without a valid causative agent, purulent discharge from the wound, abdominal pain or uterine tenderness $(4,5)$.

\section{Objectives}

Cesarean section is an important factor associated with postpartum infection and increases the risk of infection by 5-20 times compared to the vaginal delivery. Therefore, the aim of this study was to evaluate the effect of oral prophylactic antibiotics after cesarean section on the incidence of SSI.

\section{Patients and Methods}

\section{Study patients}

In this randomized single-blinded controlled clinical trial study, 462 women with emergency cesarean section who were eligible for intervention were divided into two groups using random allocation software. Intervention was oral administration of cefalexin $500 \mathrm{mg}$ every 6 hours and clindamycin $300 \mathrm{mg}$ every 6 hours for 48 hours after cesarean section $(n=231)$. Control group did not take any medication out of routine drugs $(n=231)$ (Figure 1$)$.

All participants were referred to the gynecology center on day $7-10$, at the time of removal of the sutures, and within 30 days after cesarean section, to assess the presence or absence of wound infection after surgery. Exclusion criteria were receiving antibiotics in addition to routine cefazolin, intraoperative atony event, placenta previa, decolonization, massive bleeding leading to blood transfusion, BMI $>35 \mathrm{~kg} / \mathrm{m}^{2}$, taking immunosuppressive drugs and corticosteroids, known underlying diseases including autoimmune, lymphoblastic, rheumatological and cardiac diseases, elective cesarean section, febrile patients at the time of hospitalization. Cesarean sections were generally performed by $4^{\text {th }}, 3^{\text {rd }}$, and $2^{\text {nd }}$ residential assistants with routine Williams techniques. In the followup of the patient, the physician who performed the wound evaluation on the 7-10 days of the suture removal was unaware of the group of people in the intervention and control group and only announced the outcome (surgical wound infection). The patient presented with pain, redness, and discharge for one month.

Demographic and clinical characteristics of the patients

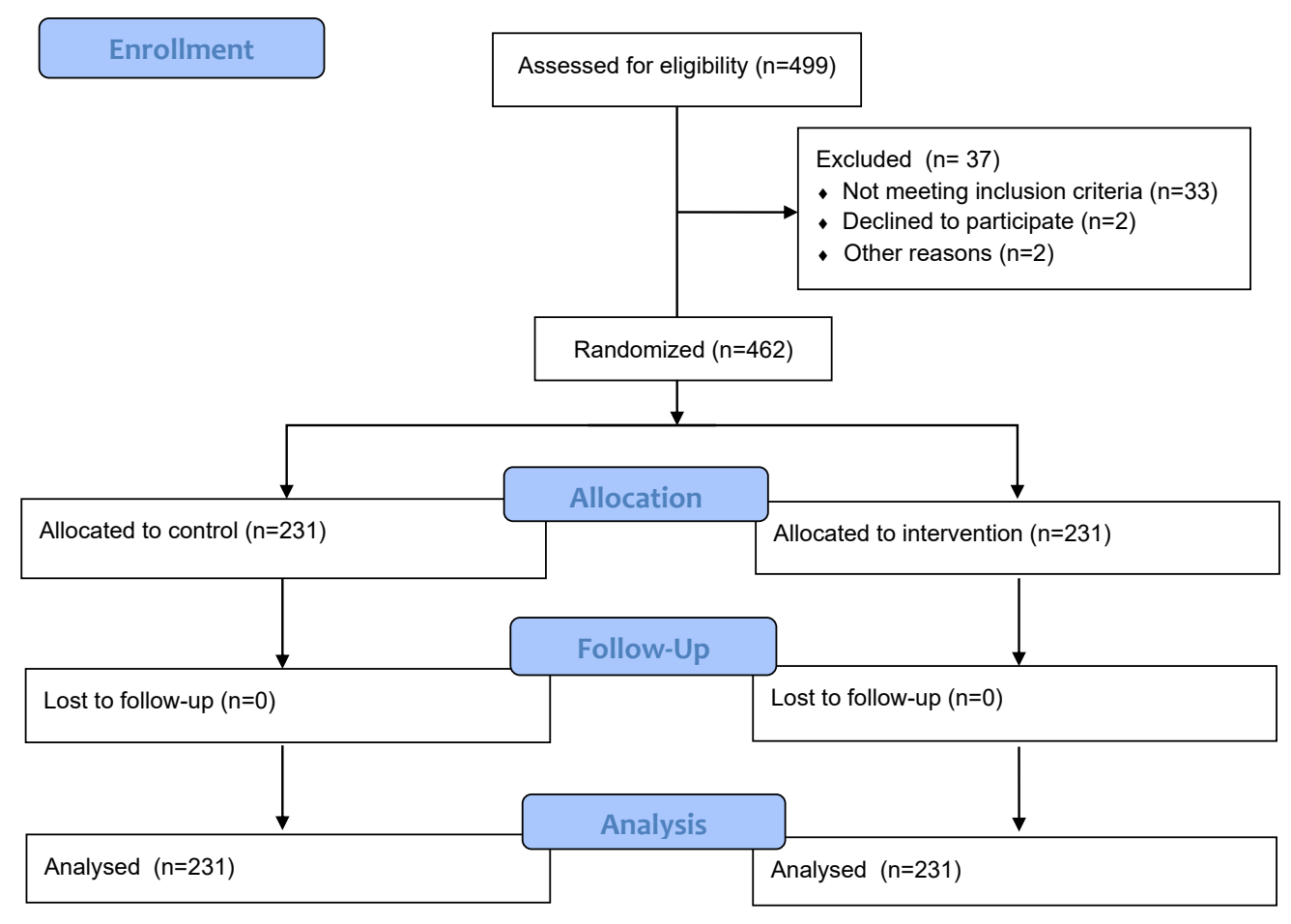

Figure 1. CONSORT Flow diagram of the study. 
in groups A and B were collected including parity, gravidity, length of stay at hospital before cesarean section, gestational age, duration of surgery, indications for cesarean section, history of cesarean section, meconium, breech and other cases, discharge, diabetes, HbAlc (as poor controlled and well controlled). The questionnaire was completed in three stages: start of the project, visit on day 7-10 and follow-up on day 30 .

\section{Data analysis}

For descriptive statistics, quantitative variables, central indices and dispersion (mean and standard deviation) were calculated, and for qualitative variables, frequency and percentage were calculated. Chi-square test and $t$ test were used to analyze the hypothesis. All statistical analyzes were performed using SPSS 21. A $P$ value less than 0.05 was considered as the significant level.

\section{Results}

The mean age of the patients in the intervention group was $29.57 \pm 5.95$ years and in the control group was $29.80 \pm 5.58$ years $(P=0.54)$. The mean parity in the intervention group was $2.45 \pm 1.18$ times and in the control group was $2.38 \pm 1.10$ times $(P=0.07)$. The mean $\mathrm{BMI}$ in the intervention group was $30 \pm 3.57 \mathrm{~kg} / \mathrm{m}^{2}$ and in the control group was $29.42 \pm 3.37 \mathrm{~kg} / \mathrm{m}^{2}(P=0.07)$. The type of incision in the intervention group was Pfannenstiel in 205 patients and midline in 26 patients (11.3\%). In the control group, 213 patients $(92.2 \%)$ had Pfannenstiel incision and 18 patients $(7.8 \%)$ had midline incision $(P=$ $0.20)$. Additionally, 68 patients $(29.4 \%)$ in the intervention group were less than 37 weeks pregnancy and 163 patients (70.6\%) were weeks $\geq 37$ pregnancy, 53 patients (22.9\%) in the control group were less than 37 weeks pregnancy and 178 patients $(77.1 \%)$ were $\geq 37$ weeks pregnancy $(P=0.11)$. The mean hematocrit before cesarean section in the intervention group was $34.30 \pm 3.56 \%$ and in the control group was $34.75 \pm 3.21 \%$. According to independent $t$ test, no significant difference between the mean hematocrit before cesarean section between the intervention and control groups was detected $(P=0.15)$. In the intervention group, 18 patients $(7.8 \%)$ had diabetes and in the control group, 15 patients $(6.5 \%)$ had diabetes. According to chisquare test, no significant difference between maternal diabetes in the two groups was seen $(P=0.58)$. Moreover, 11 patients $(4.8 \%)$ in the intervention group had multiple pregnancies and 8 patients (3.5) \%) had multiples pregnancy in the control group $(P=0.48$; Table 1$)$.

In the control group, no cesarean section infections in 186 patients $(80.5 \%)$, skin infections in 31 patients (13.4\%), subcutaneous infections in 10 patients (4.3\%) and surrounding organs became infected in 4 patients (1.7\%) after cesarean section. There was a significant difference between infection at the surgical site after cesarean section between the control and intervention groups $(P=0.001$; Table 2).
Table 1. Distribution of frequency of demographic and clinical characteristics of mothers undergoing cesarean section by group

\begin{tabular}{|c|c|c|c|}
\hline Variable & $\begin{array}{l}\text { Control } \\
\text { No. }(\%) \\
\end{array}$ & $\begin{array}{c}\text { Intervention } \\
\text { No. (\%) }\end{array}$ & $P$ value \\
\hline \multicolumn{4}{|l|}{ Age group (y) } \\
\hline$<20$ & $11(4.8)$ & $9(3.9)$ & \multirow{3}{*}{0.89} \\
\hline $20-34$ & $162(70.1)$ & $162(70.1)$ & \\
\hline$\geq 35$ & $58(25.1)$ & $60(26)$ & \\
\hline \multicolumn{4}{|l|}{ Parity } \\
\hline 1 & 39 (16.9) & $44(19)$ & \multirow{3}{*}{0.63} \\
\hline $2-4$ & $179(74.9)$ & $164(71)$ & \\
\hline$\geq 5$ & $19(8.2)$ & $23(10)$ & \\
\hline $\mathrm{BMI}\left(\mathrm{kg} / \mathrm{m}^{2}\right)$ & $29.42 \pm 3.37$ & $30.00 \pm 3.57$ & 0.07 \\
\hline \multicolumn{4}{|l|}{ Incision type } \\
\hline Pfannenstiel & $213(92.2)$ & $205(88.7)$ & \multirow[t]{2}{*}{0.20} \\
\hline Midline & $18(7.8)$ & $26(11.3)$ & \\
\hline \multicolumn{4}{|l|}{ Gestational age (wk) } \\
\hline$<37$ & $53(22.9)$ & $68(29.4)$ & \multirow[t]{2}{*}{0.11} \\
\hline$\geq 37$ & $178(77.1)$ & $163(70.6)$ & \\
\hline Hematocrit (\%) & $34.75 \pm 3.21$ & $34.30 \pm 3.56$ & 0.15 \\
\hline \multicolumn{4}{|l|}{ Surgery time (h) } \\
\hline$<1$ & $224(97)$ & $223(96.5)$ & \multirow{2}{*}{0.79} \\
\hline$\geq 1$ & $7(3)$ & $8(3.5)$ & \\
\hline Diabetes of mothers & $15(7.1)$ & $18(7.8)$ & 0.58 \\
\hline Multiple pregnancy & $8(3.5)$ & $11(4.8)$ & 0.48 \\
\hline
\end{tabular}

Table 2. Comparison of the frequency of superficial infection at the surgical site after cesarean section by group

\begin{tabular}{lccccc}
\hline \multirow{2}{*}{ Group } & \multicolumn{4}{c}{ Surgical site infection after cesarean section } & \multirow{2}{*}{ No. (\%) } \\
\cline { 2 - 5 } & Organ & Subcutaneous & Skin & $\begin{array}{c}\text { Without } \\
\text { infection }\end{array}$ \\
\hline Intervention & $0(0)$ & $2(0.9)$ & $13(5.6)$ & $216(93.5)$ & $231(100)$ \\
Control & $4(1.7)$ & $10(4.3)$ & $31(13.4)$ & $186(80.5)$ & $231(100)$ \\
Total & $4(0.9)$ & $12(2.6)$ & $44(9.5)$ & $402(87)$ & $462(100)$ \\
\hline
\end{tabular}

Regarding cesarean section infection in the intervention group based on parity, 4 patients $(22.2 \%)$ were in the study group and 14 patients $(77.8 \%)$ in the control group with one parity and cesarean section infection $(P=0.002)$. In the group of 2-4 parity, 6 patients (18.2\%) in the intervention group and 27 patients $(81.8 \%)$ were infected in the control group $(P=0.001)$. In the group of parity $\geq 5$, five patients $(55.6 \%)$ in the intervention group and 4 patients $(44.4 \%)$ in the control group had cesarean section infection $(P=0.62$; Table 3).

Regarding cesarean section infection based on incision type, in the Pfannenstiel incision, 12 patients in the intervention group (21.8\%) and 43 patients (78.2\%) in the control group had cesarean section infection. There was a significant difference between the type of Pfannenstiel incision and cesarean section infection $(P=0.01)$. In midline incision, there were three patients $(60 \%)$ in the intervention group and 2 patients (40\%) in the control group. There was no significant difference between cesarean section infection and midline incision according to Fisher's exact test $(P=0.96$; Table 4$)$. 
Of 56 patients with duration of surgery of less than one hour, 14 patients (25\%) in the intervention group and 42 patients $(75 \%)$ in the control group had infection. According to chi-square statistical test, a significant difference between the duration of surgery and cesarean section infection in the two groups was observed $(P=0.001)$. Of 4 patients with $\geq 1$ hour duration of surgery, one patient $(25 \%)$ in the study group and three patients (75\%) in the control group had infection. According to Fisher's exact test, no significant difference between the duration of surgery and cesarean section infection between the two groups in the category of surgery duration surgery more than 1 hour was found $(P=0.23$; Table 5$)$.

Regarding cesarean section infection based on maternal diabetes, of 48 diabetic patients with wound infection, 12 patients $(25 \%)$ were in the intervention group and 36 patients $(75 \%)$ were in the control group. Of 381 diabetic patients without wound infection, 201 patients $(52.8 \%)$ were in the intervention group and 180 patients (47.2\%) were in the control group. According to chi-square test, a significant difference between diabetes and cesarean section infection between the two groups was detected $(P=0.001$; Table 6).

\section{Discussion}

The prevalence of SSI in studies is up to $11 \%$. Mostly in developing countries, the estimated burden of infections such as SSI on the health care system seems high; however

Table 3. Cesarean section infection based on parity by group

\begin{tabular}{llccc}
\hline Parity & & $\begin{array}{c}\text { Control } \\
\text { No. (\%) }\end{array}$ & $\begin{array}{c}\text { Intervention } \\
\text { No. (\%) }\end{array}$ & P value \\
\hline \multirow{2}{*}{1} & With infection & $14(77.8)$ & $4(22.2)$ & \multirow{2}{*}{0.002} \\
& Without infection & $25(38.5)$ & $40(61.5)$ & \\
\multirow{2}{*}{$2-4$} & With infection & $27(81.8)$ & $6(18.2)$ & \multirow{2}{*}{0.001} \\
& Without infection & $146(48)$ & $58(52)$ & \\
\multirow{2}{*}{$\geq 5$} & With infection & $4(44.4)$ & $5(55.6)$ & \multirow{2}{*}{0.62} \\
& Without infection & $15(45.5)$ & $18(54.5)$ & \\
\hline
\end{tabular}

Table 4. Incidence of cesarean section infection by type of incision

\begin{tabular}{llccc}
\hline \multirow{2}{*}{ Incision type } & & $\begin{array}{c}\text { Control } \\
\text { No. }(\%)\end{array}$ & $\begin{array}{c}\text { Intervention } \\
\text { No. (\%) }\end{array}$ & \multirow{2}{*}{ P value } \\
\hline \multirow{2}{*}{ Pfannenstiel } & With infection & $43(87.2)$ & $4(21.8)$ & \multirow{2}{*}{0.01} \\
& Without infection & $170(46.8)$ & $193(53.2)$ & \\
\multirow{2}{*}{ Midline } & With infection & $2(40)$ & $3(60)$ & \multirow{2}{*}{0.96} \\
& Without infection & $16(41)$ & $23(59)$ & \\
\hline
\end{tabular}

Table 5. Incidence of cesarean section infection by duration of surgery

\begin{tabular}{llccc}
\hline \multicolumn{2}{l}{ Duration of surgery } & $\begin{array}{c}\text { Control } \\
\text { No. }(\%)\end{array}$ & $\begin{array}{c}\text { Intervention } \\
\text { No. (\%) }\end{array}$ & P value \\
\hline \multirow{2}{*}{$<1$ hour } & With infection & $42(75)$ & $14(25)$ & \multirow{2}{*}{0.001} \\
& Without infection & $182(46.5)$ & $209(53.5)$ & \\
$\geq 5$ hour & With infection & $3(75)$ & $1(25)$ & \multirow{2}{*}{0.23} \\
& Without infection & $4(36.4)$ & $7(63.6)$ & \\
\hline
\end{tabular}

Table 6. Incidence of cesarean section infection by diabetes of mothers

\begin{tabular}{lllll}
\hline Diabetes & $\begin{array}{l}\text { Control } \\
\text { No. }(\%)\end{array}$ & $\begin{array}{l}\text { Intervention } \\
\text { No. }(\%)\end{array}$ & $\begin{array}{l}\text { Total } \\
\text { No. (\%) }\end{array}$ & \multirow{2}{*}{$\boldsymbol{P}$ value } \\
\hline With infection & $36(75)$ & $12(25)$ & $48(100)$ & \\
Without infection & $180(47.2)$ & $201(52.8)$ & $381(100)$ & 0.001 \\
\hline Total & $216(50.3)$ & $213(49.7)$ & $429(100)$ & \\
\hline
\end{tabular}

it is not well estimated. In developing countries, SSI affects two-thirds of patients undergoing any surgery. Cesarean section is an important factor associated with postpartum infection and increases the risk of infection by 5-20 times compared to vaginal delivery (2). In our study, a significant difference regarding cesarean section infection between the two groups of use or non-use of oral prophylactic antibiotics was detected.

Findings of our study showed that 15 patients (6.5\%) in the intervention group and 45 patients $(19.5 \%)$ in the control group had a cesarean section infection $(\mathrm{P}=0.001)$. Patients receiving prophylactic antibiotics after surgery had significantly fewer deep infections $(\mathrm{P}<0.05)$. In a study conducted by Wodajo et al (2), 70.8\% of the patients had superficial infections, $26 \%$ had deep infections which are consistent with the results of our study. In a study by Valent et al, the prevalence of wound infection was generally $10.9 \%$. They reported that wound infection in the cephalexin and metronidazole treatment group was $6.4 \%$ and in the control group was $15.4 \%$ and there was a significant difference between SSI after cesarean section between the control and intervention groups $(P=0.001)$ (6).

The results of the above study are completely similar to our study. In a study by Skjeldestad et al, the rate of SSIs in the patients receiving prophylactic antibiotics after cesarean section was less (7). The results of the mentioned study were consistent with our study. In our study, the highest percentage of cesarean section infection was observed in the age group over 40 years, especially in the control group (mothers who did not receive prophylactic antibiotics). In the study by Boggess et al, there was no significant difference between maternal age and wound infection in both intervention and control groups (8).

In a study by Wodajo et al (2), the duration of hospitalization before cesarean section and rupture of membranes was an important factor in the development cesarean section infection, which was consistent with the results of our study. A study by Gelaw et al (1) also showed that mothers who were hospitalized more than 24 hours before cesarean section and had ruptured membranes before cesarean section had a higher chance of cesarean section wound infection, which was consistent with the results of our study.

In our study, the highest percentage of cesarean section infection was higher in mothers who underwent cesarean section by 2 th and 3 rd surgery assistants compared to surgeries performed by 4 th assistants. This finding was 
consistent with the results of a study by Wodajo et al, which showed that cesarean section infection was more common in young professionals. In a study by Novelia et al (9), the type of incision as an external factor led to infection at the site of cesarean section, therefore a significant difference between the intervention and control groups regarding infection with the type of incision was seen. In our study, the rate of cesarean section infection in diabetic mothers was significantly higher than in mothers without diabetes.

In the study by Novelia et al (9), maternal diabetes was one of the effective factors in increasing the rate of cesarean section infection. The study by Zuarez-Easton et al (10) revealed that intraoperative measures including an experienced surgeon team, the type of wound incision as an important factor in the prevalence, prevention and management measures can reduce wound infection after cesarean section. The incidence of cesarean section infection was significantly correlated with the type of wound incision and surgeon team.

Valent et al (6) showed a significant difference in the rate of wound infection in 13 women treated with cephalexin and metronidazole compared to 31 women receiving placebo and suggested the administration of prophylactic antibiotics after cesarean section to be effective in reducing the incidence of cesarean section infection.

\section{Conclusion}

Our study showed that the administration of prophylactic antibiotics plays an important role in reducing the incidence of cesarean section infection. It is recommended that the administration of prophylactic antibiotics be prescribed to mothers undergoing cesarean section. In this study, type of incision, skill of the surgeon, premature rupture of the membranes before cesarean section, duration of hospitalization before cesarean section and diabetes were the most important factors for SSI. Mothers with risk factors for diabetes, rupture of the membranes, leakage longer than 18 hours should take prophylactic antibiotics regularly as prescribed by the surgeon.

\section{Study limitations}

Lack of taking antibiotics by patients or not referring in time for postoperative evaluation were our main limitations. We tried to increase the accuracy of the answers by gaining their trust and designing accurate questions. It was not possible to use placebo in the control group due to the problems of placebo preparation, and on the other hand, placebo had practically no effect on the diagnosis of wound infection by the physician.

\section{Authors' contribution}

NZV, FB and SV designed the study. SV, FB and ZY performed the experiments. SV and NZV collected data from patients and helped in performance of experiments. ZY and SV prepared the primary draft after analysis. All authors read and signed the final paper.

\section{Ethical issues}

This study is a randomized controlled trial registered in the Thailand Registry of Clinical Trials (identifier: TCTR20201204002, http://www. clinicaltrials.in.th/index.php?tp=regtrials \& menu=trialsearch\&smenu=fulltext\&task $=$ search\&task $2=$ view $1 \& i d=7120$.

The study was reviewed and approved by the committee of Urmia University of Medical Sciences (IR.UMSU.REC.1397.323). Before entering the study, written consent was obtained from all patients and sufficient information about the objectives of the plan and how to implement it and review the consequences and benefits of the intervention (eg, side effects) for patients were described. Moreover, this study was extracted from the residency thesis of Naghmeh Zand Vakili, at the department of gynecology of this university. Accordingly, ethical issues (including plagiarism, data fabrication, double publication) have been completely observed by the authors.

\section{Funding/Support}

The authors would like to thank the vice chancellor of deputy research for financial support and the patients participating in this study (Grant \#1663).

\section{References}

1. Gelaw KA, Aweke AM, Astawesegn FH, Demissie BW, Zeleke LB. Surgical site infection and its associated factors following cesarean section: a cross sectional study from a public hospital in Ethiopia. Patient Saf Surg. 2017 Jun 12;11:18. doi: 10.1186/ s13037-017-0131-3.

2. Wodajo S, Belayneh M, Gebremedhin S. Magnitude and Factors Associated With Post-Cesarean Surgical Site Infection at Hawassa University Teaching and Referral Hospital, Southern Ethiopia: A Cross-sectional Study. Ethiop J Health Sci. 2017;27(3):283-290. doi: 10.4314/ejhs.v27i3.10.

3. Kawakita T, Landy HJ. Surgical site infections after cesarean delivery: epidemiology, prevention and treatment. Matern Health Neonatol Perinatol. 2017 Jul 5;3:12. doi: 10.1186/ s40748-017-0051-3.

4. Saeed KB, Greene RA, Corcoran P, O'Neill SM. Incidence of surgical site infection following caesarean section: a systematic review and meta-analysis protocol. BMJ Open. 2017 Jan 11;7(1):e013037. doi: 10.1136/bmjopen-2016-013037.

5. Warshak CR, Valent A. Intrapartum factors association with surgical site infection in the obese following cesarean delivery, and the ability of prophylactic antibiotics to reduce morbidity. Am Obst Gynecol. 2017;216(1):S57. doi: 10.1016/j. ajog.2016.11.967.

6. Valent AM, DeArmond C, Houston JM, Reddy S, Masters HR, Gold A, et al. Effect of Post-Cesarean Delivery Oral Cephalexin and Metronidazole on Surgical Site Infection among Obese Women: A Randomized Clinical Trial. JAMA. 2017 Sep 19;318(11):1026-1034. doi: 10.1001/jama.2017.10567.

7. Skjeldestad FE, Bjørnholt JV, Gran JM, Erisken HM. The effect of antibiotic prophylaxis guidelines on surgical-site infections associated with cesarean delivery. Int J Gynaecol Obstet. 2015 Feb;128(2):126-30. doi: 10.1016/j.ijgo.2014.08.018.

8. Boggess KA, Tita A, Jauk V, Saade G, Longo S, Clark EA, et al. Cesarean Section Optimal Antibiotic Prophylaxis Trial Consortium. Risk Factors for Postcesarean Maternal Infection in a Trial of Extended-Spectrum Antibiotic Prophylaxis. Obstet Gynecol. 2017;129(3):481-485. doi: 10.1097/ AOG.0000000000001899.

9. Novelia S, Sia WS, Songwathana P. Surgical Site Infection among Women Post Cesarean Section: An Integrative Review. Nurse Med J. 2017;7(1):46-55. doi: 10.14710/nmjn.v7i1.15127

10. Zuarez-Easton S, Zafran N, Garmi G, Salim R. Postcesarean wound infection: prevalence, impact, prevention, and management challenges. Int J Womens Health. 2017 Feb 17;9:81-88. doi: 10.2147/IJWH.S98876. 